\title{
Molecular Epidemiology of
} mcr-Encoded Colistin Resistance in Enterobacteriaceae From Food-Producing Animals in Italy Revealed Through the EU Harmonized Antimicrobial Resistance Monitoring

\section{OPEN ACCESS}

Edited by:

Axel Cloeckaert, Institut National de la Recherche Agronomique (INRA), France

Reviewed by: Isabelle Kempf, Agence Nationale de Sécurité Sanitaire de l'Alimentation, de l'Environnement et du Travail (ANSES), France Séamus Fanning, University College Dublin, Ireland

*Correspondence: Antonio Battisti antonio.battisti@izslt.it

Specialty section: This article was submitted to Antimicrobials, Resistance and Chemotherapy, a section of the journal Frontiers in Microbiology

Received: 21 March 2018 Accepted: 18 May 2018 Published: 12 June 2018

Citation:

Alba $P$, Leekitcharoenphon $P$,

Franco $A$, Feltrin $F$, lanzano $A$, Caprioli A, Stravino F, Hendriksen RS, Bortolaia V and Battisti A (2018) Molecular Epidemiology of mcr-Encoded Colistin Resistance in Enterobacteriaceae From

Food-Producing Animals in Italy Revealed Through the EU Harmonized Antimicrobial Resistance Monitoring. Front. Microbiol. 9:1217. doi: 10.3389/fmicb.2018.01217
Patricia Alba ${ }^{1}$, Pimlapas Leekitcharoenphon ${ }^{2}$, Alessia Franco ${ }^{1}$, Fabiola Feltrin ${ }^{1}$, Angela lanzano ${ }^{1}$, Andrea Caprioli' ${ }^{1}$, Fiorentino Stravino ${ }^{1}$, Rene S. Hendriksen², Valeria Bortolaia ${ }^{2}$ and Antonio Battisti ${ }^{1 *}$

1 Department of General Diagnostics, National Reference Laboratory for Antimicrobial Resistance, Istituto Zooprofilattico Sperimentale del Lazio e della Toscana, Rome, Italy, ${ }^{2}$ WHO Collaborating Centre for Antimicrobial Resistance in Foodborne Pathogens and Genomics and European Union Reference Laboratory for Antimicrobial Resistance, National Food Institute, Technical University of Denmark, Kongens Lyngby, Denmark

Colistin resistance by mobilisable $\mathrm{mcr}$ genes has been described in bacteria of food-animal origin worldwide, which has raised public health concerns about its potential foodborne transmission to human pathogenic bacteria. Here we provide baseline information on the molecular epidemiology of colistin-resistant, mcr-positive Escherichia coli and Salmonella isolates in food-producing animals in Italy in 20142015. A total 678, 861 and 236 indicator E. coli, Extended Spectrum Beta-Lactamase (ESBL)/AmpC-producing E. coli, and Salmonella isolates, respectively, were tested for colistin susceptibility. These isolates were collected according to the EU harmonized antimicrobial resistance monitoring program and are representative of at least 90 and $80 \%$ of the Italian poultry (broiler chickens and turkeys) and livestock (pigs and bovines < 12 months) production, respectively. Whole genome sequencing by Illumina technology and bioinformatics (Center for Genomic Epidemiology pipeline) were used to type 42 mcr-positive isolates by PCR. Colistin resistance was mainly observed in the ESBL/AmpC E. coli population, and was present in 25.9, 5.3, 6.5, and 3.9\% of such isolates in turkeys, broilers, pigs, and bovines, respectively. Most colistin-resistant isolates (141/161, 87.5\%) harbored genes of the mcr-1 group. mcr-1 was also detected in a small proportion of Salmonella isolates (3/146, 2.0\%) in turkeys. Additional $\mathrm{mcr}$ types were mcr-3 in four ESBL-producing E. coli from bovines, and two mcr-4 in $\operatorname{ESBL}(n=1)$ and indicator $E$. coli $(n=1)$ from pigs and bovines. We describe notable diversity of $\mathrm{mcr}$ variants with predominance of mcr-1.1 and mcr-1.2 on conjugative IncX4 plasmids in E. coli and in Salmonella serovars Typhimurium, Newport, Blockley from turkey. A new variant, $m c r-1.13$ was detected in the chromosome in $E$. coli in turkey and pig isolates. Additionally, we describe mcr-3.2 and mcr-4.3 in E. coli from 
bovines, and mcr-4.2 in E. coli from pigs. These findings elucidate the epidemiology of colistin resistance in food-producing animals in Italy along with its genetic background, and highlight the likelihood of $\mathrm{mcr}$ horizontal transfer between commensal bacteria and major food-borne pathogens (Salmonella) within the same type of productions. Thorough action and strategies are needed in order to mitigate the risk of $\mathrm{mcr}$ transfer to humans, in a "One Health" perspective.

Keywords: epidemiology, colistin resistance, $\operatorname{mcr}$ genes, whole genome sequencing, food-producing animals, Italy, E. coli, Salmonella

\section{INTRODUCTION}

Colistin is a polymyxin classified among the Highest Priority Critically Important Antimicrobials for human medicine by the World Health Organization (WHO), and it is considered a last resort antimicrobial for the treatment of infections by carbapenem-resistant Enterobacteriaceae in humans (Poirel et al., 2017).

In 2015 , colistin resistance mediated by $m c r-1$, a phosphoethanolamine transferase gene located on a transferable plasmid was first reported in Escherichia coli from animals, food and patients from China (Liu et al., 2016). Since then, at least 32 countries from the five continents have found mcr-1 in E. coli isolates from different sources including humans, animals and foods (Xavier et al., 2016).

In Europe, the presence of $m c r-1$ was first detected in E. coli from poultry meat and humans in Denmark (Hasman et al., 2015). Subsequently, this gene was found in Enterobacteriaceae from different sources in almost all European countries, including Italy (Battisti, 2016; Cannatelli et al., 2016). In 2016, a new $m c r$ gene, $m c r-2$, was described in E. coli in calves and piglets in Belgium (Xavier et al., 2016), followed by the description of three additional mobile colistin resistance genes in 2017, namely $\mathrm{mcr}-3$ (Yin et al., 2017) in E. coli isolated from pig in China, $m c r-4$ (Carattoli et al., 2017) in Salmonella and E. coli from pigs in Italy, Spain and Belgium, and mcr-5 (Borowiak et al., 2017) in Salmonella Paratyphi B from poultry and environmental sources from Germany.

At present, eleven mcr-1 variants (KP347127, KX236309, KU934208, KY041856, KY283125, KY352406, KY488488, KY683842, KY964067, KY853650, and LC337668), one mcr-2 variant (LT598652), six mcr-3 variants (KY924928, NPZH01000177, FLXA01000011, MF463699, MG214533, and MG489958), three $m c r-4$ variants (MF543359, MG581979, and ERS1801979) and mcr-5 (KY807921) have been described in Enterobacteriaceae according to GenBank records (last accessed 21st February 2018).

It is clear that the epidemiology of transferable $m c r$-mediated colistin resistance is evolving rapidly and timely information on prevalence and molecular epidemiology of $\mathrm{mcr}$-positive isolates is needed to enhance surveillance, and implement measures to prevention and to control further spread of colistin resistance. In the European Union (EU), the harmonized antimicrobial resistance (AMR) monitoring and reporting in poultry and livestock, which includes colistin susceptibility testing in E. coli and Salmonella, ensures that prevalence of colistin-resistant bacteria in a representative proportion of the food-animal population is reported from each Member State (MS) (Decision 2013/652/EU). However, the lack of molecular data limits the epidemiologic monitoring of colistin resistance (Schrijver et al., 2017).

The aim of this study is to determine the prevalence of colistin resistance, and the molecular epidemiology of $\mathrm{mcr}$ mediated colistin resistance genes and their genetic environment in commensal E. coli, Extended Spectrum Beta-Lactamase (ESBL)/AmpC-producing E. coli, and Salmonella spp. in foodanimals in Italy in 2014-2015.

\section{MATERIALS AND METHODS}

\section{Study Design, Sample Collection, Isolation, and Identification of Bacterial Cultures}

Study design and sampling were performed according to Decision 2013/652/EU ${ }^{1}$, which mandates each EU Member State (MS) to collect caecal content samples from different epidemiological units of poultry flocks (broiler chickens, fattening turkeys), fattening pigs and bovines $<12$ months at slaughter.

Samples were collected from broiler chickens $(n=300)$ and fattening turkeys $(n=300)$ in 2014, and from fattening pigs $(n=304)$ and bovines $<12$ months $(n=223)$ in 2015 (Table 1). The regional stratification of samples represented at least 90 and $80 \%$ of the Italian poultry (broiler chickens and turkeys) and livestock (pigs and bovines $<12$ months) production, respectively.

In addition, 558 and 709 samples from fattening turkey and broiler chicken flocks, respectively, were collected within the voluntary national Salmonella monitoring framework (Decision 2013/652/EU) in 2014 (Table 1). In compliance with Decision 2013/652/EU, E. coli isolation and identification, were performed according to the EURL-AR protocols ${ }^{2}$, whereas Salmonella spp., isolation, identification and serotyping were performed according to the ISO 6579:2002/Amd 1:2017 protocols.

\footnotetext{
${ }^{1}$ http://eur-lex.europa.eu/legal-content/EN/TXT/HTML/?uri=CELEX: 32013D0652\&from $=$ IT

${ }^{2}$ https://www.eurl-ar.eu/CustomerData/Files/Folders/21-protocols/276_esblampc-cpeprotocol-version-caecal-january2017-version4.pdf
} 
TABLE 1 | Colistin-resistance and mer genes in Escherichia coli and Salmonella sp. from caecal samples in animal primary productions, Italy, $2014-2015$.

\begin{tabular}{|c|c|c|c|c|c|c|c|c|c|c|c|}
\hline $\begin{array}{l}\text { Animal } \\
\text { production }\end{array}$ & Bacterial species & Year & $\begin{array}{l}\text { Samples } \\
\text { (n) }\end{array}$ & $\begin{array}{l}\text { Isolates } \\
\text { tested } \\
\text { (n) }\end{array}$ & $\begin{array}{c}\text { Colistin R } \\
\text { (n; \%) }\end{array}$ & $\begin{array}{c}\text { MIC range } \\
\text { mg/L } \\
\text { (mode) }\end{array}$ & $\begin{array}{c}\text { mcr-1 } \\
\text { pos. } \\
\text { (n) }\end{array}$ & $\begin{array}{c}\text { mcr-2 } \\
\text { pos. } \\
\text { (n) }\end{array}$ & $\begin{array}{c}\text { mcr-3 } \\
\text { pos. } \\
\text { (n) }\end{array}$ & $\begin{array}{c}\text { mcr-4 } \\
\text { pos. } \\
\text { (n) }\end{array}$ & $\begin{array}{c}\text { mcr-5 } \\
\text { pos. } \\
\text { (n) }\end{array}$ \\
\hline \multirow{3}{*}{$\begin{array}{l}\text { Fattening } \\
\text { turkeys }\end{array}$} & Indicator E. coli & 2014 & 300 & 170 & 39 (22.9\%) & $4-16(8)$ & 38 & 0 & 0 & 0 & 0 \\
\hline & ESBL/AmpC E. coli & 2014 & 300 & 224 & 58 (25.9\%) & $4-16(4)$ & 58 & 0 & 0 & 0 & 0 \\
\hline & Salmonella spp. & 2014 & 558 & 146 & $12(8.3 \%)$ & $4-16(4)$ & 3 & 0 & 0 & 0 & 0 \\
\hline \multirow[t]{3}{*}{ Broiler chickens } & Indicator E. coli & 2014 & 300 & 170 & 9 (5.9\%) & $4-8(4)$ & 8 & 0 & 0 & 0 & 0 \\
\hline & ESBL/AmpC E. coli & 2014 & 300 & 244 & $13(5.3 \%)$ & $4-16(4)$ & 11 & 0 & 0 & 0 & 0 \\
\hline & Salmonella spp. & 2014 & 709 & 90 & 0 & - & - & - & - & 0 & 0 \\
\hline \multirow[t]{2}{*}{ Fattening pigs } & Indicator E. coli & 2015 & 304 & 168 & $1(0.6 \%)$ & $4(4)$ & 1 & 0 & 0 & 0 & 0 \\
\hline & ESBL/AmpC E. coli & 2015 & 304 & 214 & $14(6.5 \%)$ & $4-8(4)$ & 13 & 0 & 0 & 1 & 0 \\
\hline \multirow{2}{*}{$\begin{array}{l}\text { Bovine animals } \\
<12 \text { months }\end{array}$} & Indicator E. coli & 2015 & 223 & 170 & $8(4.7 \%)$ & $4-8(4)$ & 5 & 0 & 0 & 1 & 0 \\
\hline & ESBL/AmpC E. coli & 2015 & 223 & 179 & 7 (3.9\%) & $4-8(4)$ & 4 & 0 & 4 & 0 & 0 \\
\hline
\end{tabular}

\section{Antimicrobial Susceptibility Testing}

Antimicrobial susceptibility was tested by minimum inhibitory concentration (MIC) determination using the broth microdilution method, and consensus 96-well microtiter plates (TREK Diagnostic Systems, Westlake, OH, United States). Antimicrobials tested, dilution ranges and interpretation of MIC values were in accordance with Decision 2013/652/EU.

\section{mcr and ESBL/AmpC Genes Screening}

The presence of $m c r$ was investigated in all isolates displaying colistin MIC above the epidemiological cut-off (i.e., $>2 \mathrm{mg} / \mathrm{L}$ ). A multiple PCR was used to detect $m c r-1, m c r-2, m c r-3, m c r-4$, and $m c r-5$ (Rebelo et al., 2018).

ESBL/AmpC-producing E. coli positive for $m c r$ were further screened for $b a_{\mathrm{CTX}-\mathrm{M}}, b l a_{\mathrm{SHV}}, b l a_{\mathrm{TEM}}, b l a_{\mathrm{OXA}}, b l a_{\mathrm{CMY}-1}$, and bla $a_{\mathrm{CMY}-2}$ using primers and PCR conditions previously described (Donati et al., 2014; Franco et al., 2015). Obtained amplicons were Sanger sequenced and analyzed as previously described (Donati et al., 2014; Franco et al., 2015).

\section{Conjugation Experiments}

Four representative E. coli and three Salmonella sp. isolates from turkeys were selected as donors. Conjugation experiments were performed as previously described (Franco et al., 2015), with the only modification regarding the MacConkey agar plates selective for transconjugants which contained $2 \mathrm{mg} / \mathrm{L}$ colistin sulfate and $100 \mathrm{mg} / \mathrm{L}$ rifampicin in this study.

\section{Whole Genome Sequencing (WGS) and Bioinformatics Analysis}

A total 42 isolates which tested $m c r$-positive by PCR, $(28$ E. coli and three Salmonella enterica isolates from turkeys, five E. coli from pigs and six E. coli from cattle), and the seven E. coli K12 isolates result of the conjugation experiments were Whole Genome Sequenced. Genomic DNA was extracted using the QIAamp DNA Mini Kit (Qiagen, Hilden, Germany) following the manufacturer's protocol. Libraries were prepared for Illumina pair-end sequencing using the Illumina (Illumina, Inc., San Diego, CA, United States) NexteraXT ${ }^{\oplus}$ Guide 150319425031942. Sequencing was performed using an Illumina platform (MiSeq or
HiSeq2000). Raw sequence data were submitted to the European Nucleotide Archive ${ }^{3}$ under study accession no.: PRJEB23728, PRJEB23778, PRJEB21546, and PRJEB26479.

Raw reads were assembled and analyzed using the pipeline from the Center for Genomic Epidemiology (CGE ${ }^{4}$, Thomsen et al., 2016), with default settings. This pipeline performed de novo assembly (Velvet based), species identification (KmerFinder 2.1), Multilocus Sequence Typing (MLST 1.6), identification of virulence (VirulenceFinder 1.2) and antimicrobial resistance genes (ResFinder 2.1), identification of plasmid incompatibility groups (PlasmidFinder 1.2) and plasmid MLST (pMLST 1.4). When identity values for $m c r$ were less than $100 \%$ in the ResFinder output, the sequence was submitted to online BLAST ${ }^{5}$ (Zhang et al., 2000) to identify the exact $\mathrm{mcr}$ variant.

Manual annotation of the contigs containing selected $\mathrm{mcr}$ variants was performed using RAST (Aziz et al., 2008), BLAST (Zhang et al., 2000) and ISfinder (Siguier et al., 2006). The contigs harboring the new $m c r$ variants were compared with reference sequences using BLAST and EasyFig (Sullivan et al., 2011). The references sequences used for comparison were CP016034, KP347127 and KY924928.

\section{RESULTS}

\section{Colistin Resistance and $m c r$ in E. coli and Salmonella From Turkeys and Broilers in 2014}

In 2014, colistin resistance was detected in $25.9 \%$ (58/224) and $5.3 \%(13 / 244)$ of the ESBL/AmpC-producing E. coli from fattening turkeys and broilers, respectively. In turkey flocks, all but two colistin-resistant E. coli were multidrugresistant (MDR) isolates, (i.e., resistant to three antimicrobial classes). In MDR ESBL/AmpC-producing E. coli population of turkey flocks, colistin resistance was associated with concurrent

\footnotetext{
${ }^{3}$ http://www.ebi.ac.uk/ena

${ }^{4}$ http://www.genomicepidemiology.org/

${ }^{5}$ https://blast.ncbi.nlm.nih.gov/Blast.cgi
} 
fluoroquinolone microbiological resistance (ciprofloxacin MIC $>0.064 \mathrm{mg} / \mathrm{L})$ in 51 of 58 isolates $(87.9 \%)$, with $35 / 58(60.3 \%)$ displaying fluoroquinolone clinical resistance $(\mathrm{MIC}>1 \mathrm{mg} / \mathrm{L}$, mode $8 \mathrm{mg} / \mathrm{L}$ ) (Supplementary Table 1).

A similar prevalence was observed among the indicator commensal E. coli, with colistin resistance occurring in $22.9 \%$ (39/170) and 5.9\% (9/170) of isolates from turkeys and broilers, respectively (Table 1). Nearly all colistin-resistant $E$. coli from turkey and broilers tested PCR-positive for $m c r-1$ independent of colistin MIC (Table 1).

In 2014 , colistin resistance was detected in $8.3 \%(12 / 146)$ and $0 \%(0 / 90)$ of Salmonella spp. isolates from fattening turkeys and broiler chickens, respectively. $m c r-1$ was detected only in three of twelve isolates, which displayed colistin MIC $=8 \mathrm{mg} / \mathrm{L}$. The remaining isolates $(n=9)$ did not yield any $m c r$ amplicon (Table 1).

\section{Colistin Resistance and $m c r$ in E. coli From Fattening Pigs and Bovines $<12$ Months in 2015}

In 2015 , colistin resistance occurred in $6.5 \%$ (14/214) and 3.9\% (7/179) of ESBL/AmpC-producing E. coli from fattening pigs and bovines $<12$ months, respectively. Among the indicator E. coli population, colistin resistance occurred in $0.6 \%(1 / 168)$ and $4.7 \%$ $(8 / 170)$ of isolates from fattening pigs and bovines $<12$ months, respectively (Table 1). All colistin-resistant E. coli from pigs tested positive for $m c r$ genes, 14 out of 15 harbored $m c r-1$ and one ESBL-producing isolate tested positive for $\mathrm{mcr}$-4 (Table 1). Only five out of eight colistin-resistant indicator E. coli from bovines yielded $m c r-1$ and one out of eight presented $m c r-4$ (Table 1). All seven ESBL/AmpC-producing E. coli from bovines yielded $m c r$ genes (Table 1). Thus, $m c r-1$ was detected in three isolates, $m c r-3$ in three isolates and both $m c r-1$ and $m c r-3$ in one isolate (Supplementary Table 1).

\section{Genomic Characterisation}

High diversity of Sequences Types (STs) was evident when studying the genome of the $28 \mathrm{E}$. coli and three Salmonella enterica isolates from turkeys, five E. coli from pigs and six E. coli from bovine $<12$ months selected for WGS. In E. coli from turkeys, 20 STs were identified. The most represented STs were ST-155 and ST-156 with four isolates each, followed by ST-744 with three isolates and ST-101 with two isolates (Supplementary Table 1). The five E. coli from pigs and six E. coli from cattle isolates presented different ST. The serotype and the STs of the three S. enterica were also different: S. Typhimurium ST-3515, S. Blockley ST-52 and S. Newport ST-45 (Supplementary Table 1).

mcr-1.1 (NG_050417.1) was present in 24 isolates: 18 E. coli and two Salmonella isolates from turkeys, three E. coli from pigs and one from bovines. mcr-1.2 (KX236309.1) was detected in eight E. coli and one Salmonella from turkeys. In addition, one E. coli isolate from turkey presented a silent mutation, C801T, and two $E$. coli isolates, one from turkey and one from fattening pigs showed a new variant (hereafter termed $m c r-1.13$ ) of $m c r-1.1$ with two non-synonymous mutations: M2V and S14G. Four E. coli from bovines $<12$ months presented a $m c r-3$ variant, identified as mcr-3.2 (NG_055523.1), and one of them presented both $m c r-1.1$ and mcr-3.2 (Supplementary Table 1). Also, one ESBL(CTX-M1)-producing E. coli isolate from pigs and one indicator $E$. coli from bovines presented $m c r-4.2$ and $m c r-4.3$, respectively.

The two E. coli isolates harboring mcr-1.13 allele, ID:14077295 from turkey and ID:15056414 from fattening pig, were genetically different, as shown by MLST (ST-69; ST-5995) and different resistance gene content (Supplementary Table 1). The virulence gene gad was detected in both isolates, but it was the only virulence-associated gene found in the pig isolate (Supplementary Table 1). Both isolates harbored the IncF replicon plasmid, but with different plasmid MLST: IncF [F1:A1:B20] and IncF [F46:A-:B42], for the turkey and the pig isolate, respectively (Supplementary Table 1).

All the nine isolates presenting $m c r-1.2$ (IDs:14087995, 14069546, 14062120, 14044802, 14091902, 14045775, 14047606, 14083136, and 14085183) were isolated from turkey. These isolates were one $S$. Blockley, and eight E. coli isolates with different STs (Supplementary Table 1). The resistance and virulence genes were variable among those isolates (Supplementary Table 1). For example, one of them (ID:14044802) was only resistant to colistin and only presented the accessory gene $m c r-1.2$, while all other isolates were MDR and ESC-R, and presented ESBL genes such as $b l a_{\mathrm{CTX}-\mathrm{M}-1}$ or $b l a_{\mathrm{TEM}-52}$ or other different betalactamase genes (Supplementary Table 1). A variety of plasmid incompatibility groups was observed including the plasmids IncFII, IncFI, IncHI2, IncI, IncN in different proportions, but all the nine isolates presented the plasmid IncX4 (Supplementary Table 1). In the isolate ID:14065450, the contig containing $m c r-1.2$ also contained the replicon plasmid IncX4 (contig 35).

The four WGS sequenced isolates harboring mcr-3.2 (IDs:15054212, 15038100, 15056874, and 15078696), all of them E. coli from bovines $<12$ months, had different STs and serotype (Supplementary Table 1). All four were MDR and presented the ESBL gene $b l a_{\mathrm{CTX}-\mathrm{M}-55}$, although always found in contigs other than those containing $m c r-3.2$. The four isolates also shared other resistance accessory genes as sul3, aac(3)-Iid, aadA2, or floR. A number of different plasmids were detected in all four isolates, with only IncF (F46:A-:B20), IncX1 and IncR detected in all of them. The contigs harboring $m c r-3.2$ did not contain any plasmid replicon type (Supplementary Table 1).

One of the $m c r$-3.2-positive E. coli (ID:15054212), presented also $m c r-1.1$. This isolate was ST-744 and contained the following plasmids: IncFIB, IncX4, IncFIC(FII), IncX1, Col156, IncR. $m c r-$ 1.1 was located in a 32,823 bp contig that included also the replicon sequence of IncX4 (Supplementary Table 1).

The $m c r-4.2$ variant was isolated from an E. coli from fattening pig (ID: 15057173-5). This isolate, ST-410, presented the following accessory resistance genes: bla $a_{\mathrm{CTX}}-\mathrm{M}-1$, tet $(B)-$ like, sul1,sul2, aadA1, aadB, aph(3')-Ic-like, strA, strB, $m p h(A)$, floR-like and qnrB42-like (Supplementary Table 1). $m c r-4.2$ and ColE10 replicon sequence were located in the same contig (contig 105; length $7699 \mathrm{bp}$ ). In addition to the ColE10 plasmid replicon, this isolate presented other plasmids (Supplementary Table 1).

The $m c r-4.3$ variant was harbored in a E. coli (ST-399) isolated from a bovine $<12$ months (ID: 15050011-1) that also 
presented other accessory resistance genes as $b l a_{\mathrm{TEM}-1 \mathrm{~B}}, \operatorname{tet}(B)$, sul1-like, sul3, dfrA1, aac(3)-IIa, aadA1, aph(3')-Ic-like, strA, strB and catA1-like (Supplementary Table 1). A variety of plasmids was found, including IncFIA, IncHI1A, IncHI1B(R27), ColE10, IncQ1, ColRNAI, p0111, and Col(MG828), but none in the same contig containing $\mathrm{mcr}-4.3$.

\section{Description of the Contig Harboring mcr-1.13}

mcr-1.13 was identified in E. coli strain 14077295 (contig 564; 10,310 bp lenght) from turkeys and in E. coli strain 15056414 (contig 27; 50,840 bp lenght) from pigs. The BLAST alignment of the two contigs showed $99 \%$ identity across the entire length of the shortest contig $(10,310 \mathrm{bp})$. No plasmid replicon was detected in any of the two contigs. $m c r-1.13$ (from nt 33,836 to nt 35,462 in contig 27,) was upstream to a PAP2 superfamily hypothetical protein (from nt 35,509 to nt 36,256). Insertion sequences (IS) were detected flanking the $\mathrm{mcr}$ cassette: upstream mcr-1.13 a truncated IS66 (from nt 33,335 to 33,413 bp and from 33,455 to $33,651 \mathrm{bp}$ ) and downstream a small fragment of IS66 (from nt 36,261 to nt 36,392) and IS110 (from nt 36,449 to nt $36,816)$. Upstream the cassette, there was the gene coding for $50 \mathrm{~S}$ ribosome-binding GTPase family protein (from nt 15,712 to $\mathrm{nt}$ 16,627 ) and downsteam the cassette, there was the coding gene of the subunit YeeA of the methylase (from nt 37,032 to nt 39,849) (Figure 1). In BLAST results, contig sequences showed 93-99\% identity to $E$. coli chromosome sequence CP016034 except for the region flanked by ISs and containing $m c r-1.13$ and PAP2 superfamily coding genes that had 99 and 100\% identity with the same region of the $m c r$ plasmid pHNSHP45(KP347127), for the $m c r$ gene and the PAP2 superfamily coding gene, respectively (Figure 1).

\section{Description of the Contig Harboring mcr-3.2}

$m c r-3.2$ was detected in E. coli isolates 15054212 (contig 77), 15038100 (contig 128), 15056874 (contig 52), and 15078696 (contig 197) measuring 3,346, 9,285, 5,921, and $5.279 \mathrm{bp}$, respectively. All contigs were $100 \%$ identical to the shortest one. Upstream and downstream $m c r-3.2$ (from 766 to 2,392 bp in contig 77) a diacylglycerol kinase gene (from 268 to 249 bp) and a gene coding for a NimC/NimA putative family protein (from 2,436 to 2,696 bp) were detected, respectively. These three genes were flanked by IS3 (from 1 to $127 \mathrm{bp}$ ) and Tn3 (from 2,943 to 3,346 bp) (Figure 2). The shortest contig harboring these genes (contig number 77) showed a 99\% identity with the pWJ1 plasmid containing $m c r-3.1$ (KY924928) region from 160,180 to $163,525 \mathrm{bp}$. The genes surrounding the cassette in our strains were not found in the pWJ1 plasmid (KY924928).

\section{Plasmid Transferability}

E. coli harboring $m c r-1.1$ (IDs:14043377 and 14065450), mcr1.2 (ID:14047606) and $m c r-1.13$ (ID:14077295), S. Typhimurium harboring $m c r-1.1$ (ID:14043372), $S$. Newport harboring $m c r-1.1$

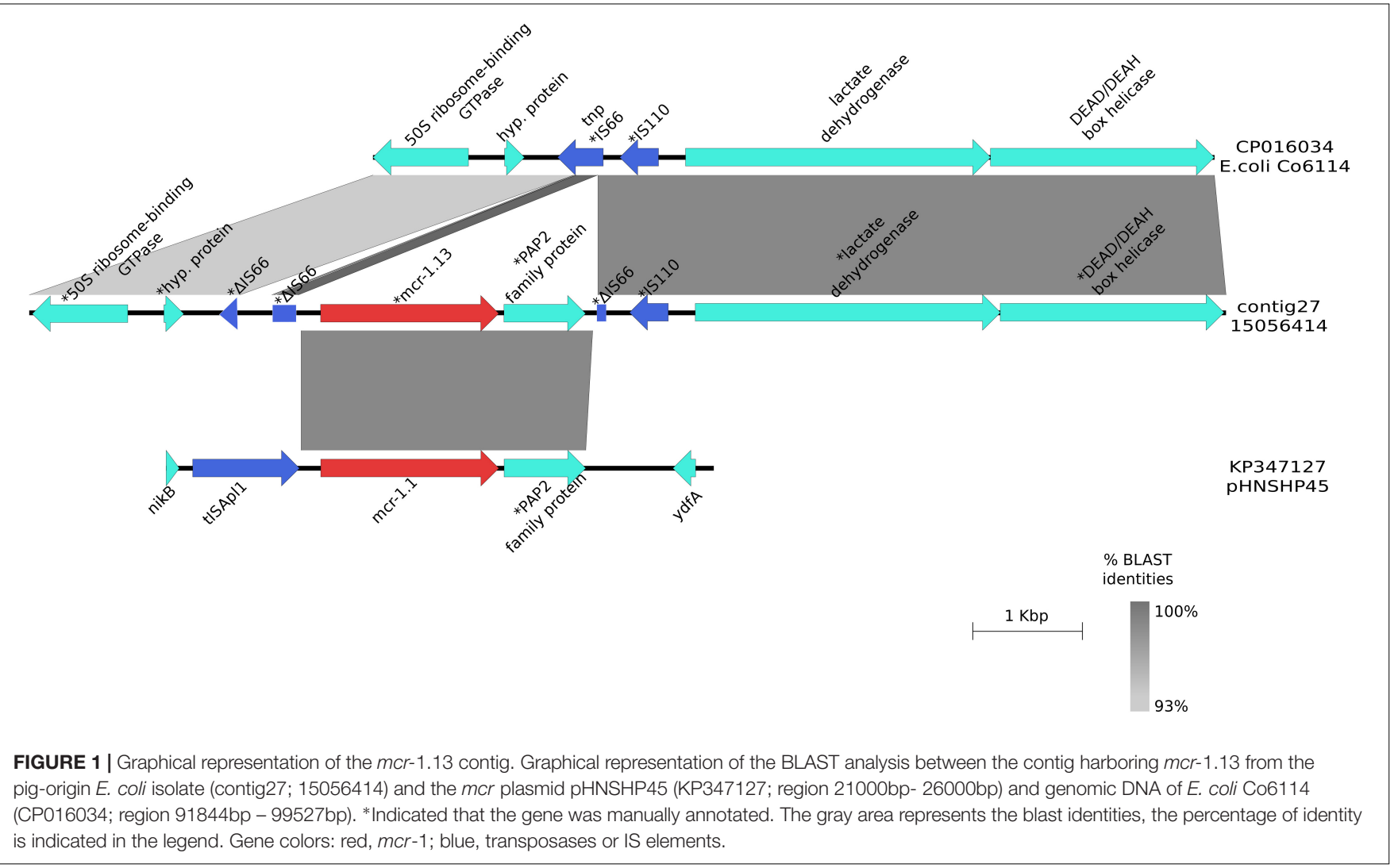




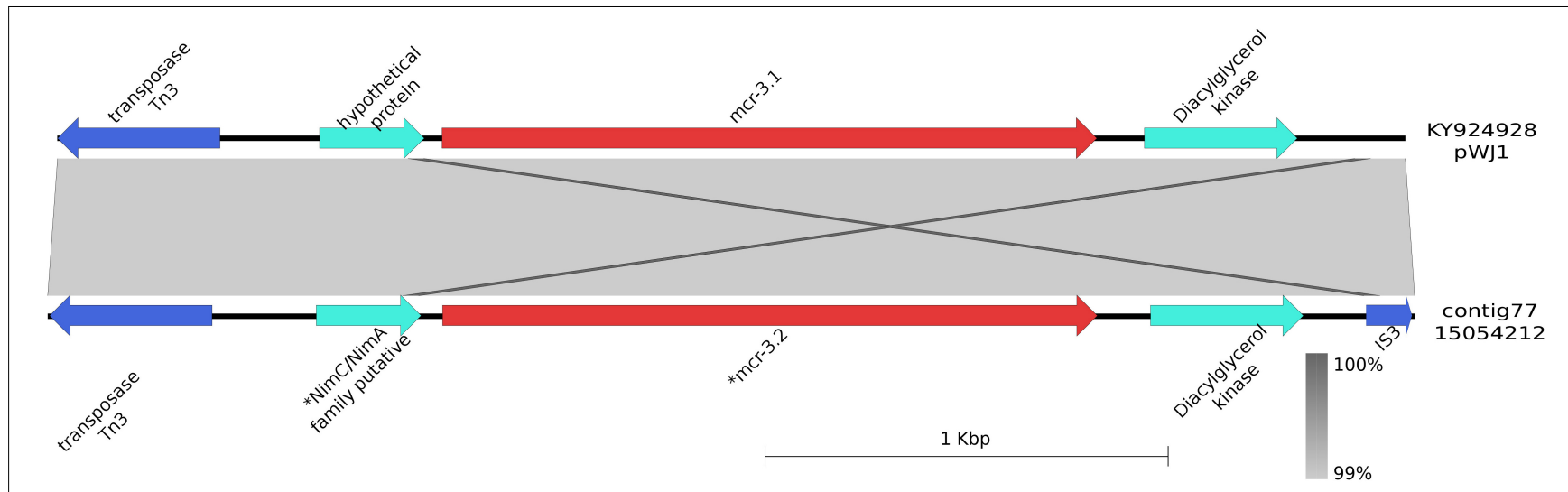

FIGURE 2 | Graphical representation of the mcr-3.2 contig. Graphical representation of the BLAST analysis between the contig harboring mcr-3.2 in the bovine-origin E. coli (contig77; 15054212) and the mcr plasmid pWJ1 (KY924928; region 160180 to 163525 bp). *Indicated that the gene was manually annotated. The gray area represents the blast identities, the percentage of identity is indicated in the legend. Gene colors: red, mcr-1; blue, transposases or IS elements.

(ID:14038647) and S. Blockley harboring mcr-1.2 (ID:14085183) were used as donors for conjugation experiments (Table 2 and Supplementary Table 1). All donors, except the mcr-1.13harboring E. coli, transferred $m c r$ by conjugation to the E. coli $\mathrm{K}-12$ recipient strain. $m c r-1.1$ and $m c r-1.2$ were carried by IncX 4 plasmids that transferred either alone or in combination with additional plasmids (Table 2 and Supplementary Table 1). One isolate (ID: 14065450) displayed in the same contig both $\mathrm{mcr}-1.1$ and the replicon of the IncX4 plasmid (contig 68).

\section{DISCUSSION}

The high diversity of transferable colistin resistance mediated by $\mathrm{mor}$ genes and alleles, quickly spreading across pathogenic enterobacteria globally (Kluytmans, 2017), is an emerging challenge for treatment of Gram-negative infections due to increased occurrence of Healthcare-Associated Extremely Drug Resistant bacterial pathogens (EARS-Net, 2015).

In the present study, we found high prevalence ( 25\%) of colistin resistance in both indicator commensal E. coli and ESBL/AmpC-producing E. coli in turkeys in Italy. In other Italian primary productions such as broilers, fattening pigs and bovines $<12$ months, colistin-resistant E. coli occurred at relatively lower, though still relevant levels.

From a (Veterinary) Public Health perspective, the spread of colistin resistance across production types is of major concern also considering that it was often associated with resistance to multiple drugs and notably also to fluoroquinolones. For instance, MDR E. coli exhibiting co-resistance to three Critically Important Antimicrobials for humans were detected in approximately $23 \%$ of turkey flocks investigated.

By using WGS, the vast diversity of $m o r$ types and variants was evident within and across the major production types. At least three $m c r-1$ alleles were identified in turkeys, pigs and bovines $<12$ months in a 2-year-period, one of which has not been previously described. The majority of isolates harbored $m c r-1.1$, but $m c r-1.2, m c r-1.13, m c r-3.2, m c r-4.2$ and $m c r-4.3$ were also detected.

The core genomes of the E. coli and Salmonella isolates harboring $m c r-1$ were quite variable as shown by diversity of MLST types, in agreement with what has been observed in different contexts previously (Hasman et al., 2015; Bernasconi et al., 2016; Cannatelli et al., 2016; Zhao et al., 2017).

Conjugation experiments and WGS data analysis confirmed that $m c r-1.1$ and $m c r-1.2$ were located on IncX4 plasmids in E. coli and Salmonella spp. from turkey. In Europe, IncX4 plasmids have been described in association with $m c r-1$ in isolates of animal origin in Belgium (Xavier et al., 2016; Garcia-Graells et al., 2017) and from humans in Italy (Di Pilato et al., 2016).

The $m c r-1.2$ gene was first described on an IncX4 plasmid in a Klebsiella pneumoniae isolated from a human sample in the Tuscany Region (Italy) (Di Pilato et al., 2016). In our study, this variant was found on IncX4 plasmids in intestinal Salmonella and E. coli spread throughout the Italian fattening turkey production. Our observations suggest the transmission of $\mathrm{mcr}$-positive IncX4 plasmids between different bacterial species, with the possibility of transmission from animals to humans, or vice versa.

The $m c r-3$ gene was first described on an IncHI plasmid (Yin et al., 2017), whereas the $m c r-3.2$ variant has been described from the whole genome shotgun sequence of a Shigella sonnei strain (NG_055523; NPZH01000177) isolated in the United States, but to our understanding, no formal description has been published to date. In our study, four ESBL/AmpC-producing E. coli isolates from bovines $<12$ months presented $m c r-3.2$. The WGS data analysis performed on these isolates did not allow us to determine the genomic location of mcr-3.2. However, only one of these isolates harbored an IncHI plasmid. A ST heterogeneity of E. coli isolates harboring the $m c r-3.2$ gene was evident. Further conjugation or transformation experiments are needed in order to elucidate if the $m c r-3.2$ found in E. coli in Italian cattle is plasmid-borne and transferable.

One E. coli from cattle was simultaneously positive for $\mathrm{mcr}$ 3.2 and $m c r-1.1$. In this isolate, $m c r-1.1$, was located on an IncX4 plasmid. An E. coli carrying both mcr-1.1 and mcr-3.2 
TABLE 2 | Genotypic traits of donors and transconjugants.

\begin{tabular}{|c|c|c|c|c|c|}
\hline \multirow[t]{2}{*}{ Strain ID } & \multirow[t]{2}{*}{ Species } & \multicolumn{2}{|c|}{ Donors } & \multicolumn{2}{|c|}{ Transconjugants (E. coli K12) } \\
\hline & & AMR genes $^{1}$ & Plasmid content ${ }^{1}$ & AMR genes $^{1}$ & Plasmid content ${ }^{1}$ \\
\hline 14043377 & E. coli & $\begin{array}{l}\text { mcr-1.1, bla TEM-52C, tet(A), } \\
\operatorname{aad} A 1, \text { dfrA1 }\end{array}$ & $\begin{array}{l}\text { IncFll, Incl1, IncFIB, p0111, } \\
\text { IncX4 }\end{array}$ & $m c r-1.1$ & $\operatorname{lncX} 4$ \\
\hline 14065450 & E. coli & $\begin{array}{l}\text { mcr-1.1, blasHv_-12, sul2, sul3, } \\
\text { aadA1, aadA2, strA, strB, } \\
\text { aph(3')-la, cmlA1 }\end{array}$ & $\begin{array}{l}\text { IncFIB, IncFII, Incl1, IncFIC, } \\
\text { IncY, IncQ1, IncX4, Col(MG828) }\end{array}$ & $m c r-1.1$ & $\operatorname{lncX} 4$ \\
\hline 14047606 & E. coli & $\begin{array}{l}\text { mcr-1.2, bla } \mathrm{CTX}-\mathrm{M}-1, \text { tet }(\mathrm{B}) \\
\text { catA1 }\end{array}$ & IncN, IncX4, Col(MG828) & $m c r-1.2$, bla $\mathrm{CTX}-\mathrm{M}-1$ & $\operatorname{IncN}, \operatorname{IncX} 4$ \\
\hline 14077295 & E. coli & $\begin{array}{l}\text { mcr-1.13, bla } 1 \mathrm{TE}-1 \mathrm{~B} \\
\text { bla }_{\mathrm{CTX}-\mathrm{M}-14}, \operatorname{tet}(\mathrm{B}), \text { sul2, strA, } \\
\operatorname{str} B, \operatorname{dfr} A 14, \operatorname{mph}(\mathrm{A})\end{array}$ & IncFII, IncFIA, IncFIB, Col156 & - & - \\
\hline 14043372 & $\begin{array}{l}\text { Salmonella } \\
\text { Typhimurium }\end{array}$ & $\begin{array}{l}m c r-1.1, \text { bla } \mathrm{TEM}-1 \mathrm{~B}, \operatorname{tet}(\mathrm{A}) \\
\operatorname{aad} 1, \operatorname{aac}(3)-I l d\end{array}$ & $\begin{array}{l}\text { Incl1, IncX4, ColRNAl, Col156, } \\
\text { ColpV }\end{array}$ & $\begin{array}{l}\text { mcr-1.1, bla } \mathrm{TEM}-1 \mathrm{~B}, \operatorname{tet}(\mathrm{A}), \\
\operatorname{aad} 1, \operatorname{aac}(3)-I l d\end{array}$ & $\begin{array}{l}\text { Incl1, ColRNAI, Col156, } \\
\text { ColpVC, IncX4 }\end{array}$ \\
\hline 14085183 & $\begin{array}{l}\text { Salmonella } \\
\text { Blockley }\end{array}$ & $\begin{array}{l}m c r-1.2, \operatorname{aph}\left(3^{\prime}\right)-1 c, \text { strA, strB, } \\
m p h(A)\end{array}$ & IncN, IncX4, Col156, ColRNAI & $m c r-1.2$ & $\operatorname{lncX} 4$ \\
\hline 14038647 & $\begin{array}{l}\text { Salmonella } \\
\text { Newport }\end{array}$ & $\begin{array}{l}\text { mcr-1.1, blaTEM-1B, tet(A), sul2, } \\
\text { strA-like, strB, dfrA14 }\end{array}$ & IncN, IncX4,ColpVC & $m c r-1.1$ & $\operatorname{lncX} 4$ \\
\hline
\end{tabular}

${ }^{1}$ Antimicrobial resistance (AMR) genes and plasmid replicons were detected using ResFinder and PlasmidFinder at http://www.genomicepidemiology.org/.

and also isolated from cattle has been recently described in Spain (Hernández et al., 2017). Interestingly, all four $m c r$-3.2-positive E. coli isolated detected in bovines $<12$ months in Italy carried the ESBL bla $a_{\mathrm{CTX}-\mathrm{M}-55}$ gene, apparently an emerging variant in veal calves in Italy. The same observation has been made by other authors (Hernández et al., 2017; Roer et al., 2017). Taken together, these features could be suggestive of a genetic linkage between the two genes. Our study, however, provides preliminary data that the mcr-3.2 and the bla $a_{\mathrm{CTX}-\mathrm{M}-55}$ genes may not be linked on the same genetic element, and that this aspect needs further investigation.

The mcr-4.2 gene was described recently from two $S$. enterica Typhimurium (monophasic variant) isolated from human samples in Italy (Carretto et al., 2018). So far, this variant has not been associated to any plasmid. In our study, the $m c r-4.2$ gene identified in a ESBL(CTX-M-1)-producing E. coli isolated from a fattening pig, was located on a ColE plasmid, exactly the same type of plasmid in which $m c r-4.1$ was described by Carattoli et al., 2017. The mcr-4.3 variant was first described from a Salmonella Kedougou isolated from pigs in Spain (Rebelo et al., 2018). In our study, $m c r-4.3$ was harbored by an indicator $E$. coli from a bovine caecal sample. All the mcr-4 variants described so far have been found in Salmonella or E. coli from animals and from humans, despite their localisation on non-conjugative plasmids, similarly to what has been found with $m c r-4.1$ by Carattoli et al. (2017).

The $m c r-1.13$ gene was located on a mobile genetic element inserted in the chromosome. The $\mathrm{mcr}$ cassette found is different from the chromosomal $\mathrm{mcr}$ cassettes described so far, because of the presence of ISs other than ISplA (Snesrud et al., 2016; Li et al., 2017). By considering the high rate of self-transferability of the IncX plasmids (Sun et al., 2017) and that IncX4 is dominant in the Italian primary productions surveyed, this finding suggests that the $\mathrm{mcr}$ cassette may have been acquired from an IncX4 plasmid which lacks ISplA insertion sequence (Bernasconi et al., 2016; Matamoros et al., 2017; Sun et al., 2017). It is likely that the insertion occurred in different moments, since the two E. coli isolates derived from two different animal productions and presented different genome content. The ability of the $\mathrm{mcr}$ cassette to jump into several types of plasmids (IncI2, IncX4, IncHI2, and IncP) or into the chromosome is highly concerning also in view of the possibility of insertion into plasmids already present in MDR isolates, or even of the creation of a new megaplasmid as occurred in Salmonella Infantis (Franco et al., 2015). This would imply that colistin resistance could be co-selected by the use of others antimicrobials. Indeed, multidrug resistance in colistin-resistant, $\mathrm{mcr}$-positive isolates is a constant feature we have observed in these population-based studies. These findings highlight how rapidly mobile genetic elements can be acquired by Enterobacteriaceae and how genes can mutate in environments with high selective pressure as that occurring in the intensive farming systems. In this respect, the selection pressure exerted by the use of colistin and the complex co-selection mechanisms triggered by the overall high use of several antibiotic classes in the meat-producing industry is likely to have played a major role. For instance, the overall exposure to antibiotics in food-producing animals in Italy in 2014 was estimated around $360 \mathrm{mg} /$ Population Correction Unit (PCU, i.e., mg per kilogram of biomass of farmed animals), and the exposure to colistin only was estimated around $29 \mathrm{mg} / \mathrm{PCU}$ (ESVAC, 2016). The mean, median and range of total sales in Europe (29 countries) were 108, 66, and $3-419 \mathrm{mg} / \mathrm{PCU}$, respectively, while 22 out of 29 countries reported sales of colistin $\leq 5 \mathrm{mg} / \mathrm{PCU}$ (range of the $29 \mathrm{EU}$ countries: $0.06-36.10 \mathrm{mg} / \mathrm{PCU}$ ). It is well known that the intensively farmed, meat-producing animals (turkeys, broilers, pigs, bovines $<12$ months) are the population categories at risk of exposure to higher amounts of colistin in modern farming systems, and the results of this study strongly support the general concept that the spread of mcr-mediated colistin resistance has been favored by the semicontinuous and high exposure to colistin in these production chains. 
Whether animals are an important source for human extraintestinal pathogenic E. coli (ExPEC) infections in humans is still a matter of debate (Bélanger et al., 2011). Few studies provide information and comparison on STs and plasmids harboring ESBLs or transferable AmpCs (Leverstein-van Hall et al., 2011) for both human and food-producing animal isolates. Although conclusive epidemiological evidence is still lacking, it has been proposed that some human ExPEC infections could arise from poultry and pig ExPEC reservoirs (Jakobsen et al., 2010). That being said, it is interesting to notice that at least one-fourth of E. coli isolates described in our study belong to the same STs as isolates associated with human ExPEC infections in Europe. In some cases (Leverstein-van Hall et al., 2011; Mavroidi et al., 2012; Brolund et al., 2014) they also share the same ESBL (e.g., ST69 and CTX-M-14, ST410 and CTX-M-1). As for colistinresistant $E$. coli, one of the $m c r-1.1$ positive isolates from turkeys here described belongs to ST131, although it lacks the virulence gene markers and ESBL generally associated with the globally spread human clinical clone. Indeed, a $m c r$-1-positive, ESBLnegative ST-131 E. coli was also described in human bloodstream infections in Italy in 2017 (Corbella et al., 2017). Overall, these observations should be taken very cautiously, since methods for genome analysis and parameters for assessing relatedness among both core genomes and accessory genomes are quickly evolving, which implies that it may be necessary to re-evaluate any earlier conclusions on relatedness or source attribution based on partial molecular characteristics, as previously shown (De Been et al., 2014).

\section{CONCLUSION}

In conclusion, harmonized cross sectional studies at slaughter like the ones implemented by the EU represent a very important tool for a deep insight into trends and emergence of antimicrobial resistance traits and patterns in major food-borne pathogens and commensal opportunistic bacteria. Especially when occurring at high prevalence, the spread of transferable colistin resistance in E. coli (both indicator commensal and ESBL/AmpC-producing isolates) is to be considered a concern per se. Additionally, as a general principle, the high spread of resistance increases the probability of transfer of specific resistance traits also to major zoonotic pathogens, such as Salmonella spp. The hypothesis that horizontal transfer, so far, has played a major role in spread of colistin resistance among bacteria in Italian meat-producing animals is supported by the observed heterogeneity of $\mathrm{mcr}$ positive E. coli. Indeed, at least in the Italian turkey productions,

\section{REFERENCES}

Aziz, R. K., Bartels, D., Best, A. A., DeJongh, M., Disz, T., Edwards, R. A., et al. (2008). The RAST Server: rapid annotations using subsystems technology. BMC Genomics 9:75. doi: 10.1186/1471-2164-9-75

Battisti, A. (2016). Antibiotic Resistance - Italy: Colistin, mcr-1, E. coli, Turkeys, 2014. Available at: http://www.promedmail.org/

Bélanger, L., Garenaux, A., Harel, J., Boulianne, M., Nadeau, E., and Dozois, C. M. (2011). Escherichia coli from animal reservoirs as a potential source of human we demonstrated that the same transferable determinant of colistin-resistance is being carried on the same conjugative plasmid in both E. coli and major Salmonella serotypes detected in the same intensive-farming industry.

For the above reasons, quick and thorough action should be taken by the farming industry and by the Competent Authorities to drastically reduce the use of colistin in food-producing animals, especially in turkeys, following the recommendations of the European Medicines Agency ( $\leq 5 \mathrm{mg} / \mathrm{PCU}$ ). EU Member States were encouraged to set stricter national targets, ideally below $1 \mathrm{mg} / \mathrm{PCU}$ colistin. We also strongly recommend reducing the overall use of all other classes of antibiotics at primary production level, in order to mitigate the effects of the complex mechanisms behind co-selection and multidrug resistance toward Critically Important Antimicrobials, in a "Consumer Protection" and a "One Health" perspective.

\section{AUTHOR CONTRIBUTIONS}

$\mathrm{PA}, \mathrm{PL}, \mathrm{AF}, \mathrm{RH}, \mathrm{VB}$, and $\mathrm{AB}$ conceived and designed the experiments. PA, PL, FF, AI, and FS performed the experiments. $\mathrm{PA}, \mathrm{AF}, \mathrm{FF}, \mathrm{AI}, \mathrm{RH}, \mathrm{VB}$, and $\mathrm{AB}$ analyzed the data. PA, PL, AF, $\mathrm{AC}, \mathrm{RH}, \mathrm{VB}$, and $\mathrm{AB}$ contributed reagents, materials, and analysis tools. $\mathrm{PA}, \mathrm{AF}, \mathrm{RH}, \mathrm{VB}$, and $\mathrm{AB}$ wrote the paper.

\section{FUNDING}

This work was supported by the project 'Establishing Next Generation sequencing Ability for Genomic analysis in Europe' (ENGAGE) co-funded by the European Food Safety Authority (EFSA, GP/EFSA/AFSCO/2015/01/CT1).

\section{ACKNOWLEDGMENTS}

The authors wish to thank Beatriz Guerra for the fruitful discussion on the manuscript, and Serena Lorenzetti, Roberta Amoruso, Carmela Buccella, Luigi Sorbara, and Roberta Onorati for outstanding technical assistance.

\section{SUPPLEMENTARY MATERIAL}

The Supplementary Material for this article can be found online at: https://www.frontiersin.org/articles/10.3389/fmicb. 2018.01217/full\#supplementary-material

extraintestinal pathogenic E. coli. FEMS Immunol. Med. Microbiol. 62, 1-10. doi: 10.1111/j.1574-695X.2011.00797.x

Bernasconi, O. J., Kuenzli, E., Pires, J., Tinguely, R., Carattoli, A., Hatz, C., et al. (2016). Travelers can import colistin-resistant Enterobacteriaceae, including those possessing the plasmid-mediated $\mathrm{mcr}-1$ gene. Antimicrob. Agents Chemother. 60, 5080-5084. doi: 10.1128/AAC.00731-16

Borowiak, M., Fischer, J., Hammerl, J. A., Hendriksen, R. S., Szabo, I., and Malorny, B. (2017). Identification of a novel transposon-associated phosphoethanolamine transferase gene, $m c r-5$, conferring colistin resistance in 
d-tartrate fermenting Salmonella enterica subsp. enterica serovar Paratyphi B. J. Antimicrob. Chemother. 72, 3317-3324. doi: 10.1093/jac/dkx327

Brolund, A., Edquist, P. J., Mäkitalo, B., Olsson-Liljequist, B., Söderblom, T., Wisell, K. T., et al. (2014). Epidemiology of extended-spectrum $\beta$-lactamase-producing Escherichia coli in Sweden 2007-2011. Clin. Microbiol. Infect. 20, O344-O352. doi: 10.1111/1469-0691.12413

Cannatelli, A., Giani, T., Antonelli, A., Principe, L., Luzzaro, F., and Rossolini, G. M. (2016). First detection of the $m c r-1$ colistin resistance gene in Escherichia coli in Italy. Antimicrob. Agents Chemother. 60, 3257-3258. doi: 10.1128/AAC. 00246-16

Carattoli, A., Villa, L., Feudi, C., Curcio, L., Orsini, S., Luppi, A., et al. (2017). Novel plasmid-mediated colistin resistance $m c r-4$ gene in Salmonella and Escherichia coli, Italy 2013, Spain and Belgium, 2015 to 2016. Euro Surveill. 22:30589. doi: 10.2807/1560-7917.ES.2017.22.31.30589

Carretto, E., Brovarone, F., Nardini, P., Russello, G., Barbarini, D., Pongolini, S., et al. (2018). Detection of $m c r-4$ positive Salmonella enterica serovar Typhimurium in clinical isolates of human origin, Italy, October to November 2016. Euro Surveill. 23:821. doi: 10.2807/1560-7917.ES.2018.23.2.17-0 0821

Corbella, M., Mariani, B., Ferrari, C., Comandatore, F., Scaltriti, E., Marone, P., et al. (2017). Three cases of mcr-1-positive colistin-resistant Escherichia coli bloodstream infections in Italy, August 2016 to January 2017. Euro Surveill. 22:30517. doi: 10.2807/1560-7917.ES.2017.22.16.30517

De Been, M., Lanza, V. F., de Toro, M., Scharringa, J., Dohmen, W., $\mathrm{Du}$, Y., et al. (2014). Dissemination of cephalosporin resistance genes between Escherichia coli strains from farm animals and humans by specific plasmid lineages. PLoS Genet. 10:e1004776. doi: 10.1371/journal.pgen.100 4776

Di Pilato, V., Arena, F., Tascini, C., Cannatelli, A., Henrici De Angelis, L., Fortunato, S., et al. (2016). $m c r-1.2$, a new $m c r$ variant carried on a transferable plasmid from a colistin-resistant KPC carbapenemase-producing Klebsiella pneumoniae strain of sequence type 512. Antimicrob. Agents Chemother. 60, 5612-5615. doi: 10.1128/AAC.01075-16

Donati, V., Feltrin, F., Hendriksen, R. S., Svendsen, C. A., Cordaro, G., GarcíaFernández, A., et al. (2014). Extended-spectrum-beta-lactamases, AmpC betalactamases and plasmid mediated quinolone resistance in Klebsiella spp. from companion animals in Italy. PLoS One 9:e90564. doi: 10.1371/journal.pone. 0090564

EARS-Net. (2015). "European centre for disease prevention and control. Antimicrobial resistance surveillance in Europe 2015," in Proceedings of the Annual Report of the European Antimicrobial Resistance Surveillance Network (Stockholm: ECDC).

ESVAC (2016). European Medicines Agency, European Surveillance of Veterinary Antimicrobial Consumption, 'Sales of Veterinary Antimicrobial Agents in 29 European Countries in 2014'. (EMA/61769/2016). Available at: http://www.ema. europa.eu/docs/en_GB/document_library/Report/2016/10/WC500214217.pdf

Franco, A., Leekitcharoenphon, P., Feltrin, F., Alba, P., Cordaro, G., Iurescia, M., et al. (2015). Emergence of a clonal lineage of multidrug-resistant ESBLproducing Salmonella infantis transmitted from broilers and broiler meat to humans in Italy between 2011 and 2014. PLoS One 10:e0144802. doi: 10.1371/ journal.pone.0144802

Garcia-Graells, C., De Keersmaecker, S. C. J., Vanneste, K., Pochet, B., Vermeersch, K., Roosens, N., et al. (2017). Detection of plasmid-mediated colistin resistance, mcr-1 and $m c r-2$ genes, in Salmonella spp. isolated from food at retail in Belgium from 2012 to 2015. Foodborne Pathog. Dis. 15, 114-117. doi: 10.1089/fpd.2017.2329

Hasman, H., Hammerum, A. M., Hansen, F., Hendriksen, R. S., Olesen, B., Agersø, Y., et al. (2015). Detection of $m c r-1$ encoding plasmid-mediated colistinresistant isolates from human bloodstream infection and imported chicken meat, Denmark 2015. Euro Surveill. 20, 1-5. doi: 10.2807/1560-7917.ES.2015. 20.49.30085

Hernández, M., Iglesias, M. R., Rodríguez-Lázaro, D., Gallardo, A., Quijada, N., Miguela-Villoldo, P., et al. (2017). Co-occurrence of colistin-resistance genes mcr-1 and mcr-3 among multidrug-resistant Escherichia coli isolated from cattle, Spain, September 2015. Euro Surveill. 22:30586. doi: 10.2807/1560-7917. ES.2017.22.31.30586

Jakobsen, L., Spangholm, D. J., Pedersen, K., Jensen, L. B., Emborg, H. D., Agersø, Y., et al. (2010). Broiler chickens, broiler chicken meat, pigs and pork as sources of ExPEC related virulence genes and resistance in Escherichia coli isolates from community-dwelling humans and UTI patients. Int. J. Food Microbiol. 142, 264-272. doi: 10.1016/j.ijfoodmicro.2010.06.025

Kluytmans, J. (2017). Plasmid-encoded colistin resistance: mcr-one, two, three and counting. Euro Surveill. 22:30588. doi: 10.2807/1560-7917.ES.2017.22.31. 30588

Leverstein-van Hall, M. A., Dierikx, C. M., Cohen Stuart, J., Voets, G. M., van den Munckhof, M. P., van Essen-Zandbergen, A., et al. (2011). National ESBL surveillance group. Dutch patients, retail chicken meat and poultry share the same ESBL genes, plasmids and strains. Clin. Microbiol. Infect. 17, 873-880. doi: 10.1111/j.1469-0691.2011.03497.x

Li, R., Yu, H., Xie, M., Chen, K., Dong, N., Lin, D., et al. (2017). Genetic basis of chromosomally-encoded $m c r-1$ gene. Int. J. Antimicrob. Agents 51, 578-585. doi: 10.1016/j.ijantimicag.2017.11.015

Liu, Y. Y., Wang, Y., Walsh, T. R., Yi, L. X., Zhang, R., Spencer, J., et al. (2016). Emergence of plasmid-mediated colistin resistance mechanism $\mathrm{mcr}$ 1 in animals and human beings in China: a microbiological and molecular biological study. Lancet Infect. Dis. 16, 161-168. doi: 10.1016/S1473-3099(15) 00424-7

Matamoros, S., van Hattem, J. M., Arcilla, M. S., Willemse, N., Melles, D. C., Penders, J., et al. (2017). Global phylogenetic analysis of Escherichia coli and plasmids carrying the $m c r-1$ gene indicates bacterial diversity but plasmid restriction. Sci. Rep. 7:15364. doi: 10.1038/s41598-017-15539-7

Mavroidi, A., Miriagou, V., Malli, E., Stefos, A., Dalekos, G. N., Tzouvelekis, L. S., et al. (2012). Emergence of Escherichia coli sequence type 410 (ST410) with KPC-2 $\beta$-lactamase. Int. J. Antimicrob. Agents 39, 247-250. doi: 10.1016/j. ijantimicag.2011.11.003

Poirel, L., Jayol, A., and Nordmann, P. (2017). Polymyxins: antibacterial activity, susceptibility testing, and resistance mechanisms encoded by plasmids or chromosomes. Clin. Microbiol. Rev. 30, 557-596. doi: 10.1128/CMR.00064-16

Rebelo, A. R., Bortolaia, V., Kjeldgaard, J. S., Pedersen, S. K., Leekitcharoenphon, P., Hansen, I. M., et al. (2018). Multiplex PCR for detection of plasmid-mediated colistin resistance determinants, $m c r-1, m c r-2, m c r-3, m c r-4$ and $m c r-5$ for surveillance purposes. Euro Surveill. 23:17-00672. doi: 10.2807/1560-7917.ES. 2018.23.6.17-00672

Roer, L., Hansen, F., Stegger, M., Sönksen, U. W., Hasman, H., and Hammerum, A. M. (2017). Novel $m c r-3$ variant, encoding mobile colistin resistance, in an ST131 Escherichia coli isolate from bloodstream infection, Denmark, 2014. Euro Surveill. 22:22846. doi: 10.2807/1560-7917.ES.2017.22.31.30584

Schrijver, R., Stijntjes, M., Rodríguez-Baño, J., Tacconelli, E., Babu Rajendran, N., and Voss, A. (2017). Review of antimicrobial resistance surveillance programmes in livestock and their meat in Europe, with a focus on antimicrobial resistance patterns in humans. Clin. Microbiol. Infect. 24, 577590. doi: 10.1016/j.cmi.2017.09.013

Siguier, P., Perochon, J., Lestrade, L., Mahillon, J., and Chandler, M. (2006). ISfinder: the reference centre for bacterial insertion sequences. Nucleic Acids Res. 34, D32-D36. doi: 10.1093/nar/gkj014

Snesrud, E., He, S., Chandler, M., Dekker, J. P., Hickman, A. B., McGann, P., et al. (2016). Model for transposition of the colistin resistance gene $\mathrm{mcr}-1$ by ISApl1. Antimicrob. Agents Chemother. 60, 6973-6976. doi: 10.1128/AAC.01457-16

Sullivan, M. J., Petty, N. K., and Beatson, S. A. (2011). Easyfig: a genome comparison visualizer. Bioinformatics 27, 1009-1010. doi: 10.1093/bioinformatics/btr039

Sun, J., Fang, L. X., Wu, Z., Deng, H., Yang, R. S., Li, X. P., et al. (2017). Genetic analysis of the IncX4 plasmids: implications for a unique pattern in the $m c r-1$ acquisition. Sci. Rep. 7:424. doi: 10.1038/s41598-017-00095-x

Thomsen, M. C., Ahrenfeldt, J., Cisneros, J. L., Jurtz, V., Larsen, M. V., Hasman, H., et al. (2016). A bacterial analysis platform: an integrated system for analysing bacterial whole genome sequencing data for clinical diagnostics and surveillance. PLoS One 11:e0157718. doi: 10.1371/journal.pone.015 7718

Xavier, B. B., Lammens, C., Ruhal, R., Kumar-Singh, S., Butaye, P., Goossens, H., et al. (2016). Identification of a novel plasmid-mediated colistin-resistance gene, $m c r-2$, in Escherichia coli, Belgium, June 2016. Euro Surveill. 21:30280. doi: 10.2807/1560-7917.ES.2016.21.27.30280

Yin, W., Li, H., Shen, Y., Liu, Z., Wang, S., Shen, Z., et al. (2017). Novel plasmidmediated colistin resistance gene mcr-3 in Escherichia coli. mBio 8:e00543-17. doi: $10.1128 / \mathrm{mBio} .00543-17$ 
Zhang, Z., Schwartz, S., Wagner, L., and Miller, W. (2000). A greedy algorithm for aligning DNA sequences. J. Comput. Biol. 7, 203-214. doi: 10.1089/ 10665270050081478

Zhao, F., Feng, Y., Lü, X., McNally, A., and Zong, Z. (2017). Remarkable diversity of Escherichia coli carrying $m c r-1$ from hospital sewage with the identification of two new mor1 variants. Front. Microbiol. 8:2094. doi: 10.3389/fmicb.2017. 02094

Disclaimer: The conclusions, findings and opinions expressed in this scientific paper reflect only the view of the authors and not the official position of the European Food Safety Authority.
Conflict of Interest Statement: The authors declare that the research was conducted in the absence of any commercial or financial relationships that could be construed as a potential conflict of interest.

Copyright (c) 2018 Alba, Leekitcharoenphon, Franco, Feltrin, Ianzano, Caprioli, Stravino, Hendriksen, Bortolaia and Battisti. This is an open-access article distributed under the terms of the Creative Commons Attribution License (CC BY). The use, distribution or reproduction in other forums is permitted, provided the original author(s) and the copyright owner are credited and that the original publication in this journal is cited, in accordance with accepted academic practice. No use, distribution or reproduction is permitted which does not comply with these terms. 